MASA/CR-1998 207376

$$
\begin{gathered}
\text { NAGI-1840 } \\
\text { IN-19-CR } \\
067803
\end{gathered}
$$

\title{
RESEARCH ON THE PROBLEM OF HIGH-PRECISION DEPLOYMENT FOR LARGE-APERTURE SPACE-BASED SCIENCE INSTRUMENTS
}

\author{
Mark S. Lake \\ NASA Langley Research Center \\ Hampton, Virginia 23681 \\ 757-864-3114 \\ Lee D. Peterson, M. Roman Hachkowski, \\ Jason D. Hinkle, and Lisa R. Hardaway \\ University of Colorado \\ Boulder, Colorado 80309 \\ 303-492-1743
}

Presented at the 1998 Space Technology \& Applications International Forum

Albuquerque, New Mexico

January 25-29, 1998 
$-$

, 


\title{
RESEARCH ON THE PROBLEM OF HIGH-PRECISION DEPLOYMENT FOR LARGE-APERTURE SPACE-BASED SCIENCE INSTRUMENTS
}

\author{
Mark S. Lake \\ NASA Langley Research Center \\ Hampton, Virginia 23681 \\ 757-864-3114
}

\author{
Lee D. Peterson, M. Roman Hachkowski, \\ Jason D. Hinkle, and Lisa R. Hardaway \\ University of Colorado \\ Boulder, Colorado 80309 \\ $303-492-1743$
}

\begin{abstract}
The present paper summarizes results from an ongoing research program conducted jointly by the University of Colorado and NASA Langley Research Center since 1994. This program has resulted in general guidelines for the design of high-precision deployment mechanisms, and tests of prototype deployable structures incorporating these mechanisms have shown microdynamically stable behavior (i.e., dimensional stability to parts per million). These advancements have resulted from the identification of numerous heretofore unknown microdynamic and micromechanical response phenomena, and the development of new test techniques and instrumentation systems to interrogate these phenomena. In addition, recent tests have begun to interrogate nanomechanical response of materials and joints and have been used to develop an understanding of nonlinear nanodynamic behavior in microdynamically stable structures. The ultimate goal of these efforts is to enable nano-precision active control of micro-precision deployable structures (i.e., active control to a resolution of parts per billion).
\end{abstract}

\section{INTRODUCTION}

NASA's Office of Space Science has recently inaugurated the Astronomical Search for Origins and Planetary Systems (Origins) Program. Between the years 2005 and 2010, this program will launch a series of extraordinary new astrophysical science instruments including the Next Generation Space Telescope (NGST, Fig. 1(a)). Also, NASA's Office of Mission to Planet Earth is studying a class of non-imaging-quality deployable telescopes for future atmospheric science missions (Fig. 1(b)). These so-called "lidar" (light direction and ranging) telescopes are to be used for the measurement of climatically important variables in the atmosphere such as water vapor, aerosol and clouds, and certain chemical constituents such as ozone. The success of these revolutionary science instruments depends on the development of many new materials and structures technologies including sub-micron-stable, actively controllable deployable structures (Peterson et al. 1996).

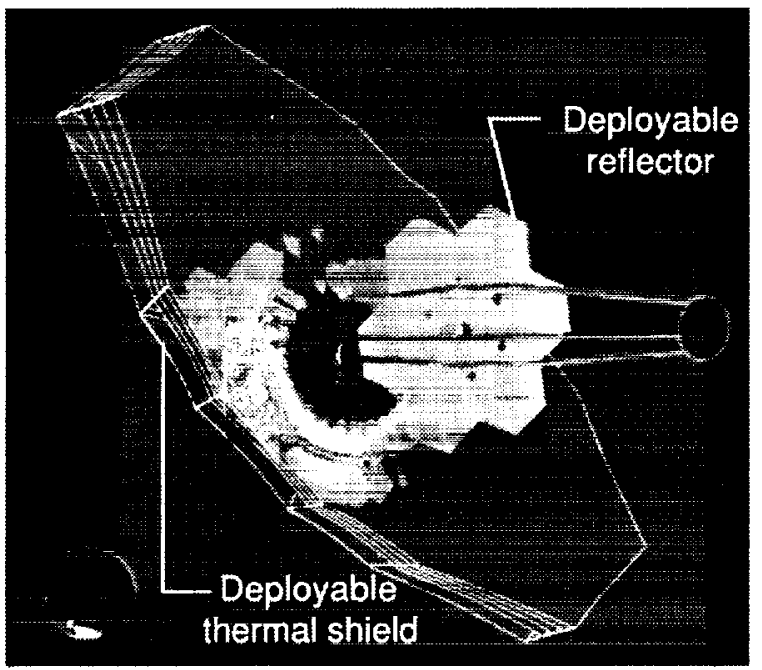

(a) Next Generation Space Telescope Concept.

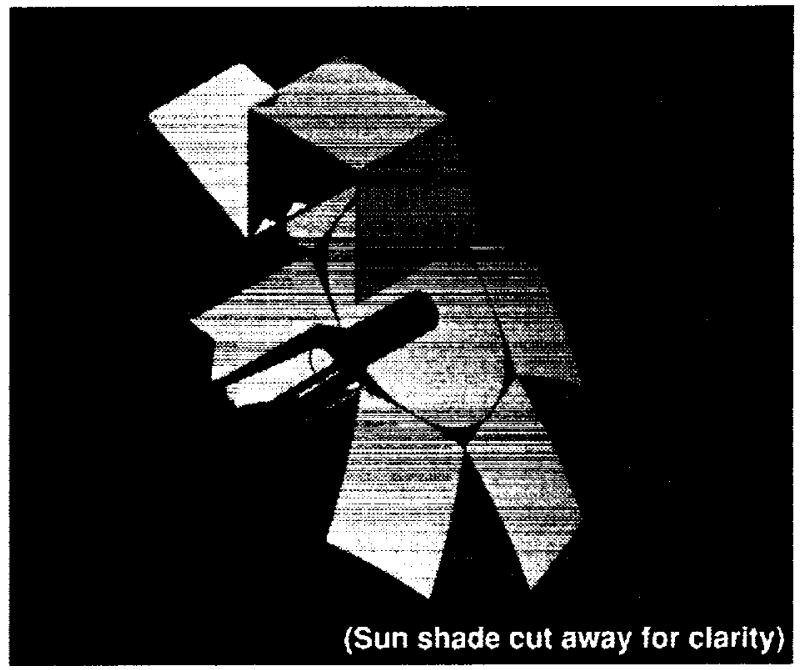

(b) Deployable Lidar Telescope Concept.

FIGURE 1. Future NASA Deployable Telescope Concepts. 
Since 1994, the University of Colorado and NASA Langley Research Center have been jointly conducting a research program to advance the state-of-the-art of high-precision deployable structures. The broad goals of this program are to enable the design of deployable structures that exhibit sufficient dimensional stability to function as metering structures for optical instruments, and to minimize the complexity and cost of such structures by maximizing passive dimensional precision and minimizing the use of active control. The specific goals of this program are: 1) to characterize experimentally and to model analytically microdynamic instabilities in deployable structures that arise from deployment mechanisms and material nonlinearities; 2) to characterize experimentally and to model analytically the micromechanical response within deployment mechanisms that gives rise to dimensional instabilities; and 3) to develop design rules for deployable structures to minimize dimensional instabilities, and to maximize deployment precision and post-deployment stability.

The present paper provides an overview of this ongoing research program. Specifically, techniques for conducting dynamic-response testing of precision deployable structures and results from some tests are presented. Dominant nonlinear microdynamic response effects derived from these tests are discussed, and the affects of these microdynamic responses on the design of active shape control systems are outlined. In addition, techniques for conducting micromechanical-response tests on deployment mechanisms and results of these tests are presented, and general guidelines for designing high-precision deployment mechanisms are derived. Finally, an overview of current research thrusts is presented.

\section{PASSIVE PRECISION VERSUS ACTIVE CONTROL OF DEPLOYABLE STRUCTURES}

The design of any lightweight precision deployable structure must involve a trade between passive precision and active control. Before explaining the issues that must be considered in performing an active-versus-passive system trade, it is useful to define explicitly two aspects to the dimensional precision of a deployed structure:

1. Deployment Precision: The error in the final deployed shape of a structure as compared to its groundmeasured shape.

2. Post-Deployment Stability: The variation in the deployed shape of a structure in response to on-orbit thermal and mechanical loads.

For moderate-precision applications (i.e., precision up to parts per hundred thousand), it is possible to satisfy the deployment precision and post-deployment stability requirements passively through proper mechanical design of the deployment mechanisms. However, high-precision applications will require on-orbit active control to meet dimensional stability requirements. To minimize the cost and complexity of active control systems, it is prudent to characterize carefully the passive deployment precision and post-deployment stability of the structure and to establish adequate, but not overly conservative, requirements for active control. For example, characterizing the passive deployment precision of a structure determines the need and requirements for an on-orbit quasi-static active control. Similarly, characterizing the post-deployment stability of a structure defines the need and requirements for highbandwidth active control.

Without a clear understanding of deployment precision and post-deployment stability, it is difficult to establish reasonable requirements for on-orbit active-control systems. Uncertainty in these requirements may lead to increased complexity (and cost) in the development of an on-orbit active-control system. Furthermore, uncertainty in the requirements leads to increased system-development risk because estimates for deployment precision and postdeployment stability requirements that are thought to be conservative might, in fact, neglect effects, such as highbandwidth microdynamics, that could substantially diminish the performance of the active system.

Thus, attaining a better understanding of passive deployment precision and post-deployment stability is necessary for the design of efficient (i.e., not overly conservative) and low-cost active control systems. While it is clear that active control cannot or should not be eliminated entirely, experience to date indicates that accurate characterization of passive deployment precision and post-deployment stability can lead to improvements in passive performance and can enable the use of efficient and low-cost active control systems. The present research program can be viewed as an effort to enable the design of precision deployable structures which are very low in cost because they incorporate a reasonable combination of passive precision and active control. 


\section{MICRODYNAMICS OF PRECISION DEPLOYABLE STRUCTURES}

The word "microdynamics" is ordinarily associated with a broad class of nonlinear structural dynamic phenomena with response magnitudes at or below the microstrain level (i.e., $10^{-6}$ times a characteristic dimension of the structure). By definition, microdynamics are nonlinear and difficult to model analytically. Furthermore, experience has shown that the inherent uncertainties associated with microdynamics leads directly to uncertainty in the postdeployment stability of a structure.

Perhaps the first question which one might ask regarding microdynamics is: "How can nonlinearities arise when motion becomes small?" This question follows from the fact that the nonlinear terms in the general governing equations of solid mechanics become negligible as deflections become small. However, these governing equations consider only geometric and material nonlinear effects which, by definition, are negligible for small motions.

For sub-microstrain levels of motion, the usual assumptions used for classical mechanics models of structures and materials are questionable. Generally, classical mechanics models exclude inhomogeneous material effects (e.g., intercrystalline slippage in metals, or fiber-matrix debonding in composite materials) and non-conservative interface mechanics (e.g., sliding, yielding, and creep that happens in friction interfaces within the structure). Experience to date indicates that non-conservative interface mechanics within deployment mechanisms dominates the microdynamics of deployable structures at the tens of microstrain level down to the nanostrain level. It is currently theorized that inhomogeneous material effects dominate the microdynamics of structures at sub-nanostrain levels. In any event, it is important to realize that the usual assumptions for classical mechanics models fail to include the effects which dominate the microdynamic response of deployable structures. However, it is incorrect to infer from this observation that microdynamic response is purely random and unpredictable.

\section{Dominant Microdynamic-Response Effects}

While our understanding and observations of microdynamic nonlinearities are incomplete, there are several effects which we currently know can affect both deployment precision and post-deployment stability. The principal effects of microdynamics which have been observed to date are the phenomena referred to as "microlurch" and the "equilibrium zone" (Warren 1996). Microlurch is a residual change in the shape of a deployed structure which occurs after a dynamic transient motion, and the equilibrium zone is the variability of a deployed structure's shape due to random microlurch response. Hence, equilibrium zone is a measure of the deployment stability of the structure.

Microlurch has been seen to occur in response to dynamic forces, such as impulses, and it is believed to occur in response to quasi-static thermal loading, although no such occurrences have been measured to date. Current models predict that microlurch is caused by a release of strain energy that is built up due to frictional interactions within the joints and latches of a deployed structure. Microlurch behavior can be either random or progressive depending on the state of the structure prior to loading. Specifically, it has been observed that just after deployment, microlurches tend to be progressive such that successive microlurches accumulate into a net, quasi-stable, shape change of the structure. In other words, it appears that structures tend to change their shape slightly after deployment to relax internal stresses built up by the deployment process. After structures have undergone a post-deployment, progressive microlurch, subsequent microlurching responses tend to be random but bounded in magnitude. The boundary of this random microlurch response is the equilibrium zone of the structure.

Repeated deployment and stowage tests of precision deployable structures have shown that deployment precision might be an order of magnitude worse than post-deployment stability once the structure has microlurched to within its equilibrium zone. The most important implication of these observations is that the equilibrium zone geometry, and NOT the initial deployed shape, determines the post-deployment stability. Furthermore, data suggest that it might be possible to design deployed structures with post-deployment stabilities to better than one part per million of the overall dimension. However, it is also important to realize that all data to date have been derived from ground tests, and the effects of gravity on the deployment repeatability and post-deployment stability of precision structures is currently unknown. 


\section{Affect of Microdynamics on the Problem of Active Control}

The existence of microlurching and an equilibrium zone, as opposed to a single equilibrium geometry, establishes the need for quasi-static shape control and possibly high-bandwidth active control for applications in which precision requirements are smaller than the size of the equilibrium zone itself. To complicate the implimentation of quasistatic shape control systems, the possibility of repeated microlurching of the structure implies that quasi-static shape adjustment might have to be repeated after fairly short intervals of time.

To complicate the implementation of high-bandwidth active shape control systems, the sudden release of strain energy during a microlurch has the potential to excite high frequency structural dynamics which can fall outside the practical bandwidth of an active control system. It is perhaps this aspect of microdynamics which is most troubling to the developer of active shape-control systems. For example, future space-based interferometers require nanometer stability up to at least $1,000 \mathrm{~Hz}$, but the nominal active control bandwidth is typically limited to several hundred Hertz. If a microlurch occurs during the formation of an image or during an astrometric measurement, it is likely that the instrument would need to be realigned and the measurement began again. Persistent events of this type would directly limit mission science data.

\section{Microdynamic Test Methodology}

Microdynamic testing is unlike conventional dynamic testing in that microdynamic-response phenomena possess random attributes that can be easily confused with random noise in the test setup. The fundamental challenges to performing accurate microdynamic tests are: 1) to control the environmental influences that are often neglected in macroscopic tests but which are significant systematic errors at microscopic or nanoscopic levels; 2) to resolve the microdynamic mechanics with "multiple witness" sensors with nanometer and nanostrain resolution; and 3) to isolate particular mechanical effects of the test apparatus from the mechanical effects of interest.

At the University of Colorado, a new facility and associated test methodologies have been recently developed which enable nanometer-resolution measurements to be made of microdynamic phenomena. The "Thermal Acoustic Stabilization Chamber" (TASC) is a 3-meter cube enclosing an optics table mounted on vibration isolation legs. The walls of the chamber are shielded for passive acoustic attenuation, and are insulated by approximately 10 inches of styrofoam for thermal isolation. Tests in the chamber typically achieve 6 micro-g vibration levels and hour-long milli-degree Kelvin thermal stability. Additionally, the facility includes high sensitivity, lightweight accelerometers, a nanometer resolution laser interferometer, and picostrain resolution strain gauges. In particular, the laser interferometer measurement path is enclosed to reduce the effect of variations in the index of refraction in air. The net result is approximately 2.5 nanometers of resolution for sampling rates ranging from quasi-static to many 10 's of kiloHertz. Such a performance level was previously only possible in a vacuum.

\section{Microdynamic Testing of a Prototype Deployable Telescope Metering Truss}

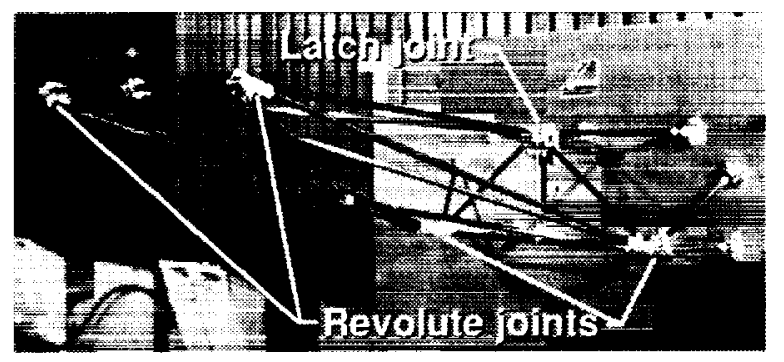

FIGURE 2. Deployable Telescope Metering Truss.

Warren (1996) presents results from tests of a deployable telescope metering truss (Fig. 2) which incorporates four precision revolute (i.e., hinge) joints and one end-of-deployment latch joint. This test article represents a portion of a metering truss that could support one reflector panel in a segmented telescope mirror (e.g., one of the six perimeter panels shown in either Fig. 1(a) or 1(b)). The metering truss is approximately $1.2 \mathrm{~m}$ in length, and exhibits deployment precision of approximately 20 microns in the position of the outboard nodes. Results from 
microdynamic testing indicate that the structure microlurches up to approximately 10 microns in response to transient disturbances subsequent to deployment. However, the structure consistently settles into an equilibrium zone of approximately 2 microns in size after progressive microlurching from its initial deployed condition.

\section{Stick-Slip Friction Model of Microlurching}

Analytical results presented from a simple two-degree-of-freedom model suggest that microlurching is an artifact of stick-slip instability due to load transfer through friction (Warren 1996). Since both the revolute joints and the latch joint possess mechanical interfaces at which load is partially transferred through friction, it is plausible that these mechanisms are responsible for microlurching in the deployable telescope metering truss.

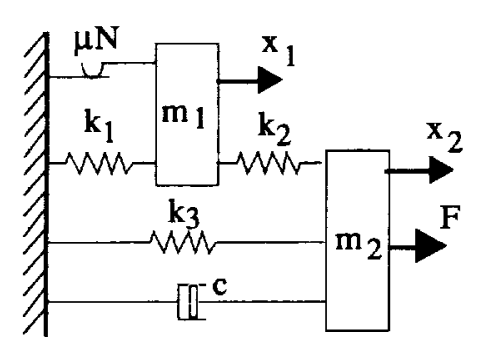

a) Model Parameters.

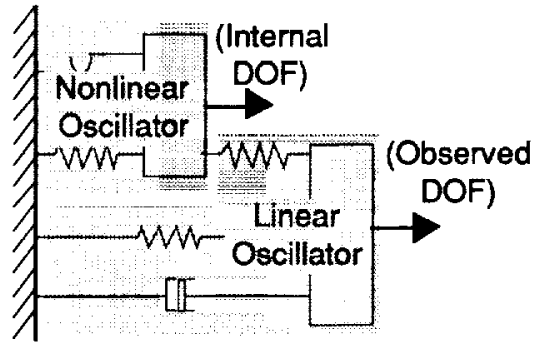

b) Dynamic-Response Interpretation.

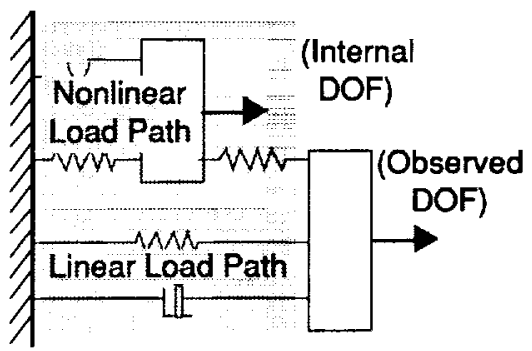

c) Mechanical-Response Interpretation.

FIGURE 3. Simple System Model from Warren (1996).

The simplified model used by Warren is shown schematically in Fig. 3 with model parameters described in Fig. 3a. In these analyses, the simplified model was used to simulate a single degree of freedom of the dynamic response of the deployable telescope metering truss shown in Fig. 2 (i.e., vertical motion of an outboard joint). The parameters of the simplified model represent modal parameters of the truss (e.g., modal masses and stiffnesses) rather than physical parameters of the truss (e.g., member masses and stiffnesses). For these dynamic-response analyses, the model can be interpreted to represent a linear oscillator coupled with a nonlinear oscillator as shown in Fig. $3 b$. The nonlinear oscillator exhibits stick-slip instabilities due to the Coulombic friction element. These instabilities give rise to microlurch motion in the total system response.

The analyses presented by Warren (1996) using this model closely agree with his extensive experimental data on the microlurching response of the deployable telescope metering truss shown in Fig. 2. These analyses reinforced laboratory findings that microlurching is random and possibly chaotic (i.e., deterministic but highly dependent on initial conditions), and dependent on excitation energy. The correlation between experimental and analytical results strongly supports the notion that microlurching is caused by stick-slip interactions within the structure.

\section{MICROMECHANICS OF DEPLOYMENT MECHANISMS}

As a consequence of lessons leamed through microdynamic testing of deployable structures, new high-precision deployment mechanisms have been developed, and substantial micromechanical response experiments have been performed. Specifically, numerous tests have been conducted to quantify the hysteretic response of these deployment mechanisms under load cycling, and efforts have been made to correlate these measured response results with analytical results from models of frictional interactions within the deployment mechanisms.

\section{Brecision Revolute Joint Concept}

Early in this research program, and with very little a priori knowledge of the relationship between nonlinear response behavior in joints and dimensional instabilities in structures, NASA Langley Research Center developed a revolute (i.e., hinge) joint concept with the hope that it would function well as a precision deployment mechanism (Lake et al. 1996). The joint concept was based on the simple premise that nonlinear mechanical response within deployment mechanisms is the fundamental limitation to post-deployment dimensional stability in a mechanically deployable structure, and therefore, a precision joint is one which responds LINEARLY to load cycling. 


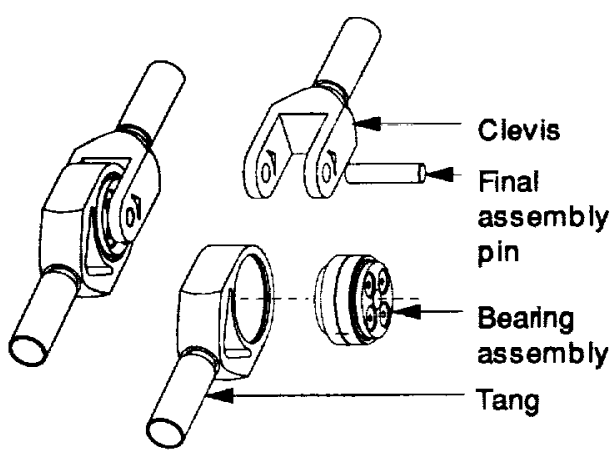

(a) Linear Revolute Joint Diagram.

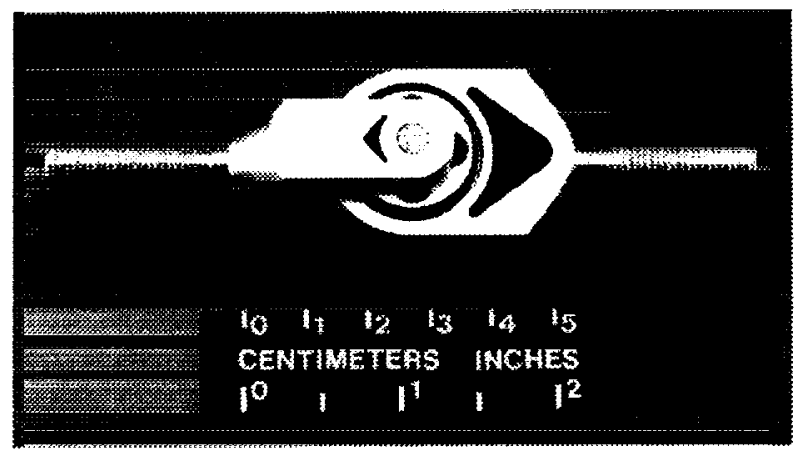

(b) Linear Revolute Joint Photograph.

FIGURE 4. Linear Revolute Joint.

The revolute joint concept (Fig. 4) represents a substantial departure from conventional pin-clevis joint designs in that it incorporates a precision, preloaded angular-contact ball bearing to allow rotation instead of a simple pin. The bearing is internally preloaded to eliminate freeplay, and the bearing diameter is maximized to minimize stiffness changes due to nonlinear interface conditions between the balls and the races. Early tests of the joint indicated that its load-cycle response is very nearly linear with less than $2 \%$ hysteresis under quasi-static load cycling.

\section{Hysteretic Response Testing}

Substantial efforts have been made to characterize the hysteretic-response behavior of the precision revolute joint, and to correlate this behavior with predictions from analytical models. Three separate studies have investigated the hysteretic load-cycling response of the precision revolute joints. Lake et al. $(1996,1997)$ presented results of extensional load-cycle tests conducted over a wide range of load-cycle magnitudes. Bullock (1996) presented results of extensional and rotational load-cycle tests conducted at low load-cycle magnitudes.

Instrumentation and Test Procedures. In each of these experimental programs, great care was taken to minimize noise and hysteresis in the load and displacement instrumentation in order to ensure accurate characterization of the hysteretic response of the specimens. In particular, fiber-optic displacement transducers were used instead of electromechanical displacement transducers because the fiber-optic instruments exhibit no hysteresis in their response. Also, the load cells used were calibrated prior to testing to ensure that their hysteretic response was insignificant.

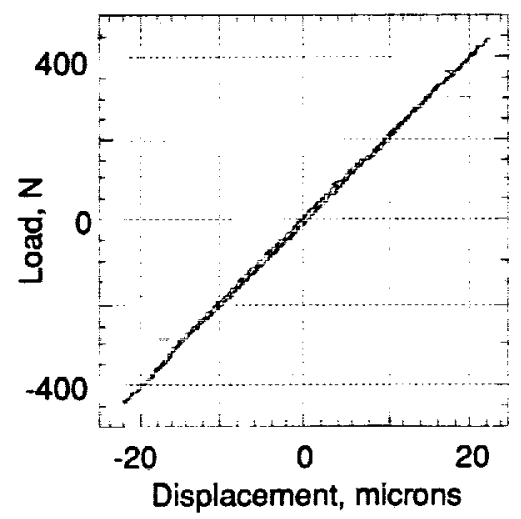

(a) Raw Response.

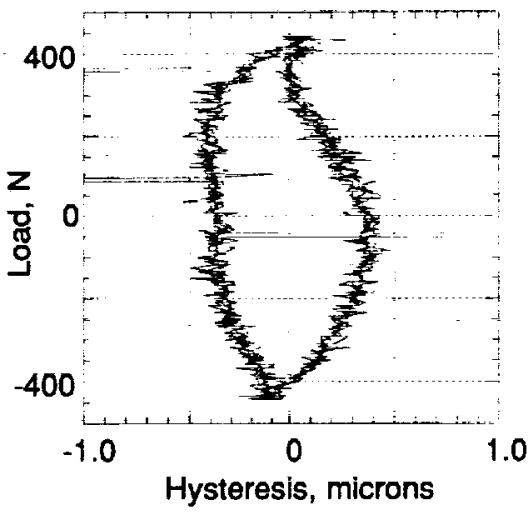

(b) Raw Hysteresis.

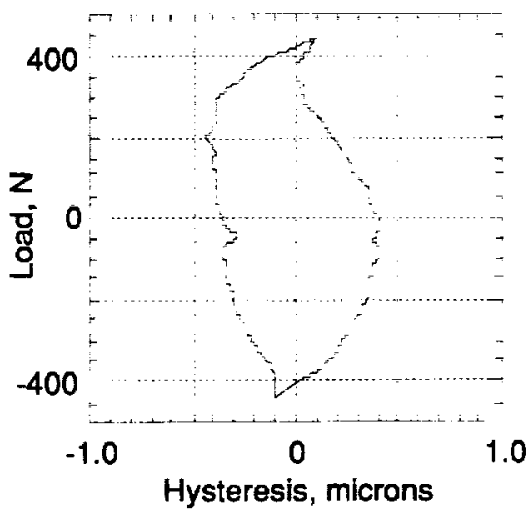

(c) Filtered Hysteresis.

FIGURE 5. Typical Load-Displacement Response from Precision Revolute Joint.

A typical load-displacement response from these tests is presented in Fig. 5 (Lake et al. 1997). The raw (i.e., unfiltered) response is presented in Fig. 5(a), and the corresponding raw hysteretic response (derived by subtracting 
the best-fit straight line from the total response) is presented in Fig. 5(b). These data illustrate that the displacement data possess more noise than the load data, and this noise is of a high-frequency nature. Consequently, substantial efforts were made during these tests to filter the displacement data channels and to improve the resolution of the displacement measurements. Several numerical filtering and averaging procedures were employed during these tests and the results are presented in Fig. 5(c). Comparing Figs. 5(b) and 5(c), it can be seen that the data filtering and averaging procedures effectively reduce displacement data noise by more than an order of magnitude leaving a filtered displacement measurement resolution of approximately $25 \mathrm{~nm}$.

General Results. Results presented in the three references (Lake et al. 1996, Lake et al. 1997, and Bullock 1996) from extensional load-cycle testing of the joint indicate that hysteretic response varies dramatically with load-cycle magnitude and internal preload within the joint. For example, data from Bullock (1996) indicate that the joint

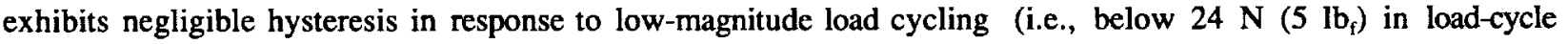
magnitude and approximately $200 \mathrm{~nm}$ of total deflection response). Data from Lake et al. (1997) indicate the hysteretic loss of the joint increases monotonically with load-cycle magnitude, and can reach values of 1 to $2 \%$ for some specimens. Together, these results indicate that friction-induced micro slippage between the internal mechanical components of the joint essentially vanishes at low load-cycle magnitudes. Therefore, within the limitations of the present tests, the joint exhibits perfect elastic response to quasi-static load-cycling at these low load-cycle magnitudes. The broader implication of this result is that structures incorporating these joints should be elastic and dimensionally stable if disturbance forces are small.

Data from Lake et al. (1997) also indicate that hysteretic energy loss occurs in BOTH the angular-contact bearing that allows rotation of the joint, and the press-fit pin that is used to affect final assembly of the joint (see Fig. 4(a)). This result might be counter-intuitive due to the fact that the press-fit pin is designed to be a highly preloaded interface that would commonly be expected to exhibit a perfectly elastic response to load cycling. Based on these data, it appears likely that nonlinear microdynamic response would even be a problem in heavily preloaded structures.

In addition to extensional load-cycle testing, Bullock conducted rotational load-cycle testing of the precision revolute joint. The motivation for these tests was to understand the nature of the hysteretic response of the joints under rotational motion, and to study the effects that this hysteretic response might have on deployment repeatablity of structures incorporating the joint. The primary result of Bullock's rotational load-cycle tests is that, unlike the case of extensional load cycling, the joint exhibits measurable hysteresis for all load-cycle magnitudes during rotational load cycling. In other words, the joint fails to be perfectly elastic in response to rotational load-cycling and structures incorporating these joints should be expected to exhibit some degree of dimensional uncertainty between successive deployments (i.e., error in deployment repeatability).

\section{Hysteretic Response Analyses}

Hachkowski (1996) correlated Bullock's rotational-response data with an analysis of the frictional resistance of the bearing to rotation. Hachkowski's analysis is based on the Todd-Johnson tribological friction model of rolling resistance within ball bearings (Todd and Johnson 1987), and includes the effects of Coulombic micro-slippage between the bearing components and material hysteretic damping. His analysis is developed using nondimensional parameters to describe bearing preload, geometry, and material properties, a formulation that facilitates parametric analysis and design optimization. The significant contribution of Hachkowski's work is that he correlated analytical predictions of rolling resistance with experimental data, and demonstrated that the error between the predicted and actual friction torques is within the uncertainty of the properties of the bearings.

More recently, Hachkowski has extended his analysis to predict the effects of micro-slippage on the response of the revolute joint under extensional load cycling. Preliminary results from this work indicate that the Coulombic micro-slippage between the bearing components leads to hysteretic loss effects similar to those found experimentally by Lake and Bullock. Furthermore, Hachkowski has demonstrated that even the simplified friction model presented in Fig. 3 predicts, qualitatively, the gross hysteretic-response behavior seen in the precision revolute joint.

The general conclusions of analyses to date are that: 1) hysteretic losses within the revolute joint are caused by localized friction-induced micro-slippage between the interfaces of the various mechanical components within the 
joint; and 2) these effects can be represented qualitatively FOR MOST, IF NOT ALL, PRECISION DEPLOYMENT MECHANISMS by the dual-load-path model suggested in Fig. 3.

\section{DESIGNING HIGH-PRECISION DEPLOYMENT MECHANISMS}

The general conclusions from Hachkowski's analyses can be applied to develop general rules for the design of high-precision deployment mechanisms. Specifically, since hysteretic response under load cycling is caused by load transfer through friction at interfaces between load-carrying components within the mechanism, it follows that a general principal to be applied in the design of high-precision deployment mechanisms is to design the load-carrying mechanical interfaces such that minimal load is transferred through friction. To understand how this design requirement might be met in practical designs consider first how friction develops at an interface.

\section{Load Transfer Across Mechanical_Interfaces}

Under the action of externally applied loads, internal load paths develop between the various mechanical components of a mechanism. As load is transmitted between adjacent mechanical components, interface stresses and localized material deformations are developed. The interface stresses and deformations can be decomposed into normal and tangential (i.e., shear) components as depicted in Fig. 6. Load transfer through normal stress at the interface involves no friction and is thus elastic. However, load transfer through shear stress at the interface involves friction, and can thus be hysteretic (i.e., inelastic) if slippage occurs.

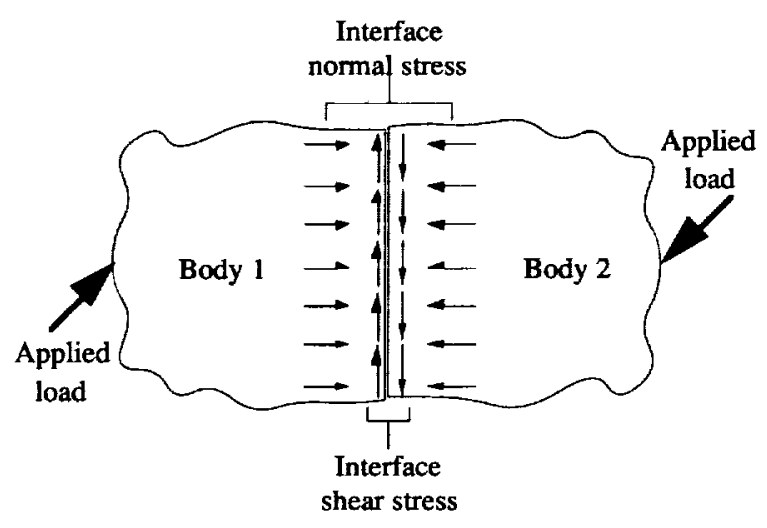

FIGURE 6. Interface Loads Between Two Mechanical Components Within a Deployment Mechanism.

The simplified model presented in Fig 3 to explain microlurching response can also be used to illustrate this dual-load-path behavior across an interface (Fig. 3(c)). This model indicates how an externally applied load, F, can be divided into elastic (i.e., normal) and inelastic (i.e., tangential) load path components for load transfer across the interface. The elastic load path involves only a single elastic element, $k_{3}$, which transmits the normal component of the externally applied load. The inelastic load path includes an elastic element, $k_{2}$, which transmits the shear component of the externally applied load from the point of application to the interface. The interface portion of the shear load path is represented by a second elastic element, $k_{1}$, in parallel with a friction element, $\mu N$. This subdivision of the shear load at the interface accounts for the fact that some of the shear load (i.e., in areas where slippage does not occur) is transferred across the interface elastically, whereas some of the shear load (i.e., in areas where slippage does occur) is transferred across the interface inelastically.

\section{General Design Rule}

To minimize the potential for inelastic load transfer, and thus to minimize the possibility of interface slippage, it is desirable to minimize shear stresses at the interfaces between mechanical components within the mechanism. $A$ number of factors affect the interface stress distribution between two elastic components in contact, and understanding which of these factors lead to the development of shear stresses at the interface is a critical step towards 
developing guidelines for the design of mechanisms which minimize interface shear stresses. However, a general rule that can be applied is:

to minimize interface shear stresses and the possibility of micro-slippage, minimize locally the tangential stiffness in the region of the interface (i.e., $k_{2}$ in Fig. 3).

In the case of the precision revolute joint presented in Fig. 4, the numerous interfaces between the balls and the races within the bearings exhibit minimal local tangential stiffness by virtue of the fact that the balls are kinematically free to roll. Hence, ball bearings are inherently good mechanisms for high precision applications.

\section{Conforming Versus Non-Conforming Interfaces}

Practically, this general rule discourages the use of "conforming" interfaces (i.e., interfaces whose geometries match over a relatively large area). Conforming interfaces involve large contact regions within which it would be very difficult to reduce or eliminate local shear stiffness. Conversely, non-conforming interfaces (e.g., a ball bearing against a race) involve relatively small regions of contact, thus lending themselves to local elastic tailoring of the structural design adjacent to the contact region to reduce shear stiffness.

\section{CURRENT RESEARCH THRUSTS}

During the past three years, the present research program has resulted in substantial advancements in understanding the problem of high-precision deployment. As a result of these advancements, it is now possible to design deployable structures that exhibit microdynamically stable behavior (i.e., dimensional stability to the level of parts per million). To further advance our understanding of the nonlinear microdynamics of these precision deployable structures and to enable active control of their geometries to the level of parts per billion, a number of new research thrusts have been recently established.

\section{Inhomogeneous Material Effects}

As mentioned previously, inhomogeneous material effects, such as intercrystalline slippage in metals, or fibermatrix debonding in composite materials might have a significant effect on nanostrain-level response in precision structures. To begin investigating these effects, thermal and mechanical stability tests have been conducted recently on a low-CTE composite strut developed for application to optical metering structures. These tests were performed in the TASC facility at the University of Colorado, and were designed to isolate nanostrain-level nonlinear response similar to microlurch. Specifically, the response of the strut to gradually varying thermal and mechanical loads was interrogated for occurrence of sudden high-frequency events such as acoustic emissions.

During these tests various combinations of axial and thermal loading were applied to the strut and its extensional response was measured to nanometers of resolution. For extensional stress up to $5 \mathrm{psi}$, temperature changes up to $20^{\circ} \mathrm{C}$, and extensional strains up to 250 nanostrain, the test specimen showed benign monotonic response to variations in loads with no evidence of the acoustic emissions.

\section{The Effects of Interface Preload on Micro-Slipoage and Load-Cycle Hysteresis}

A common misconception regarding the design of deployment mechanisms is that preload prevents slippage in a joint or latch if the load does not exceed the Coulombic limit. In fact, recent test results have shown that this notion is not the case, and that significant presliding slippage (also known as microslip) occurs in preloaded interfaces. Preload therefore does not mitigate microdynamic effects.

To investigate the phenomenon of microslippage across a preloaded interface, and to develop a better understanding of the relationship between interface preload and microdynamic response effects such as microlurch, a new test apparatus has been constructed recently at the University of Colorado. The objective of this apparatus is to test the nanostrain-level response of an interface across which load is being transferred through friction. To date, no data have been found in the literature to describe such behavior, and the present test is viewed as a benchmark effort in this area. 


\section{Systematic Methodology for Spatial Localization of Microdynamic Events}

An effort is currently underway to develop test and analysis techniques to isolate the "origin" of microdynamic events within deployable structures (i.e., the specific joint within the structure which is microslipping). In support of this effort, microdynamic tests will be conducted on a prototype deployable reflector truss and a deployable interferometer boom. The microdynamic response of these structures to a variety of static and dynamic thermal and mechanical loads are to be studied at nanometer resolution. To isolate the origin of microdynamic events, the measured response will be compared with a predicted response generated using linear models. An important feature of this approach is that it also includes the "microdynamic error" in a mathematical form which is convenient for control system design.

\section{On-Orbit Microdynamic Experiments}

One of the most critical questions in microdynamic research is the effect of gravity (in both joints and materials) on the microdynamic response of structures. To answer this question, the Micron Accuracy Deployment Experiments (MADE) facility is being developed. Intended as a permanent facility on the International Space Station, MADE is scheduled for installation in 2002. It is being developed as part of NASA's Engineering Research and Technology (ERT) program. The broad goal of MADE is to provide a capability to perform multiple, longduration microdynamic experiments on-orbit, and to correlate the on-orbit test results with ground measurements to determine the effects of gravity on microdynamic behavior.

\section{Defining Passive Limits for Deployment Repeatability and Post-Deployment Stability}

NASA Langley Research Center is currently planning the design, fabrication, and testing of a 2.1-m-diameter deployable lidar telescope mirror test article for ground microdynamic testing (Fig. 1(b)). The test article will incorporate recent advancements in precision deployment mechanisms, and recent advancements in low-areal-density and low-CTE composite reflector panels and structures. The test article is intended to help define the practical limits of passive deployment repeatability and post-deployment stability. The rationale for selecting a lidar telescope application as the focus of this test article design effort is that the dimensional precision requirements for lidar telescopes are on the order of parts per million - a level that might be attainable without the use of active control. Current plans are for the test article to be completed and the microdynamic testing to begin during 1998.

\section{SUMMARY}

Prior to the present research program, experience indicated that traditional deployment mechanisms inherently limit the dimensional stability of deployable structures to the level of approximately one millimeter over a one meter span (i.e., dimensional stability to parts per thousand.) The present research program has resulted in general guidelines for the design of high-precision deployment mechanisms, and tests of prototype deployable structures incorporating these mechanisms have shown microdynamically stable behavior (i.e., dimensional stability to parts per million). These advancements have resulted from the identification of numerous heretofore unknown microdynamic and micromechanical response phenomena, and the development of new test techniques and instrumentation systems to investigate these phenomena. In addition, tests have been begun recently to investigate the nanomechanical response of materials and joints and to develop an understanding of the nonlinear nanodynamic behavior of microdynamically stable structures. The ultimate goal of these efforts is to enable nano-precision active control of micro-precision deployable structures (i.e., active control to a resolution of parts per billion).

\section{Acknowledgment}

The work presented herein and conducted at the University of Colorado has been jointly funded by the NASA Langley Research Center (Grant No. NAG1-1840) and the Jet Propulsion Laboratory (Contract No. 960896).

\section{References}

Bullock, Steven J. (1996), "Identification of the Nonlinear Micron-Level Mechanics of Joints for Deployable Precision Space Structures," Ph.D. Dissertation, University of Colorado. 
Hachkowski, M. R. (1996), "Friction Model of a Revolute Joint for a Precision Deployable Spacecraft Structure," presented at the 37th AIAA/ASME/ASCE/AHS/ASC Structures, Structural Dynamics, and Materials Conference, Salt Lake City, UT, April 15-17, 1996, AIAA Paper No. 96-1331.

Lake, Mark S. et al. (1996), "A Revolute Joint with Linear Load-Displacement Response for Precision Deployable Structures," presented at the 37th AIAA/ASME/ASCE/AHS/ASC Structures, Structural Dynamics, and Materials Conference, Salt Lake City, UT, April 15-17, 1996, AIAA Paper No. 96-1500.

Lake, Mark S. et al. (1997), "Experimental Characterization of Hysteresis in a Revolute Joint for Precision Deployable Structures," presented at the 38th AIAA/ASME/ASCE/AHS/ASC Structures, Structural Dynamics, and Materials Conference, Kissimmee, FL, April 7-10, 1997, AIAA Paper No. 97-1379.

Peterson, L. D. et al. (1996), "Micron Accurate Deployable Antenna and Sensor Technology for New-MillenniumEra Spacecraft," presented at the 1996 IEEE Aerospace Applications Conference, Snowmass, CO, February, 1996.

Todd, M.J. and Johnson, K.L. (1987); "A Model for Coulomb Torque Hysteresis in Ball Bearings," International Journal of Mechanical Science, 29, 1987, pp. 339-354.

Warren, Peter A. (1996), "Sub-Micron Non-Linear Shape Mechanics of Precision Deployable Structures," Ph.D. Dissertation, University of Colorado. 
. 